\title{
Adrenal malignant melanoma masquerading as a pheochromocytoma in a patient with a history of a multifocal papillary and medullary thyroid carcinoma
}

\author{
Maria E. Barmpari, ${ }^{1}$ Christos Savvidis, ${ }^{1}$ Anastasia D. Dede, ${ }^{1}$ Haridimos Markogiannakis, ${ }^{2}$ \\ Christina Dikoglou, ${ }^{3}$ Paraskevi Xekouki, ${ }^{4}$ Constantine A. Stratakis, ${ }^{4}$ Manouras Andreas, ${ }^{2}$ \\ Sofia Malaktari-Skarantavou ${ }^{1}$
}

${ }^{1}$ Department of Endocrinology and Metabolism, Hippokration Hospital, Athens, ${ }^{2}$ Department of Endocrine Surgery, First Department of Propaedeutic Surgery, Hippokration Hospital, Athens Medical School, University of Athens, ${ }^{3}$ Department of Pathology, Hippokration Hospital, Athens, Greece; ${ }^{4}$ Section of Endocrinology and Genetics, Program on Developmental Endocrinology Genetics, Eunice Kennedy Shriver National Institute of Child Health and Human Development, National Institutes of Health, Bethesda, Maryland, USA

\begin{abstract}
OBJECTIVE: Adrenal masses usually represent benign and nonfunctional adrenal adenomas; however, primary or metastatic malignancy should also be considered. Discovery of an adrenal mass needs further evaluation in order to exclude malignancy and hormonal secretion. We present a rare case of a possibly primary adrenal malignant melanoma with imaging and biochemical features of a pheochromocytoma. CASE REPORT: A 61-year-old male farmer was referred for evaluation of a mass in the right supraclavicular region and a left adrenal lesion. The patient had a history of a multifocal papillary and medullary thyroid carcinoma. Laboratory tests revealed increased 24hour urinary dopamine and also increased serum calcitonin and neuron specific enolase. A pathology report of the resected right supraclavicular mass and left adrenal showed a malignant melanoma. CONCLUSION: This is a case of a possibly primary adrenal malignant melanoma with imaging and biochemical features of a pheochromocytoma. Although this case is very rare and there are rigid diagnostic criteria for the diagnosis of primary adrenal melanoma, it underlines the fact that the differential diagnosis of a dopamine secreting adrenal mass should include primary or metastatic malignant melanoma in order to determine the best diagnostic approach for the patient and select the most appropriate surgical management.
\end{abstract}

Key words: Adrenal melanoma, Dopamine, Metastasis, Pheochromocytoma, Primary

Address for correspondence:

Maria E. Barmpari, MD, MSc, 114 Vas. Sofias Av., 11527

Ampelokipoi, Athens, Greece; Tel.: +30 6946208218,

Fax: +30 2132088665, E-mail: barbari_maria@yahoo.com

Received: 04-12-2015, Accepted: 09-12-2015

\section{INTRODUCTION}

Pheochromocytomas are catecholamine-secreting tumors arising from the adrenal medulla. They present either as solitary tumors (unilateral or multifocal) or 
in conjunction with other endocrine neoplasms. When concurrent with medullary thyroid carcinoma they can be a major component of multiple endocrine neoplasia type 2 (MEN2), whose genetic basis is a germline mutation in the RET proto-oncogene. ${ }^{1}$ Discovery of an adrenal mass requires further evaluation in order to exclude malignancy and hormonal secretion. ${ }^{2,3}$ Metastasis of cancer cells to the adrenals is not very uncommon, because of the rich blood supply of these glands, and reflects an advanced stage of several types of malignancies such as lung, renal and colon cancer., ${ }^{4,5}$

Malignant cutaneous melanoma is a particularly life-threatening type of skin cancer that is derived from melanocytes normally present in the epidermis. ${ }^{6,7}$ While adrenal gland metastases can be seen in the context of metastatic malignant melanoma, primary adrenal melanoma is extremely rare and only a small number of cases have been published in the literature. ${ }^{8-10}$ Herein we report a unique case of a possibly primary malignant adrenal melanoma, which had biochemical and radiological findings suggestive of a pheochromocytoma, in a patient with a history of a medullary and papillary thyroid carcinoma.

\section{CASE DESCRIPTION}

A 61-year-old male farmer was referred for evaluation of a mass in the right supraclavicular region and a left adrenal lesion. There was nothing noteworthy in the patient's family medical history. The patient had a history of total thyroidectomy with central and right lateral cervical lymph node dissection three years previously. The histopathological examination was positive for medullary carcinoma and multifocal papillary thyroid carcinoma, both metastatic to regional lymph nodes. He had received radioiodine ablation therapy and was under thyroxin suppression therapy. Further imaging studies were negative. Genetic testing for RET proto-oncogene mutation revealed a common polymorphism in exon (c.2071 G>A/p.G691S) and a synonymous variation in exon 15 (c.2712 C $>\mathrm{G} / \mathrm{p}$. S904S). During follow-up, due to the neck swelling, he underwent a neck ultrasound and a 2-(18F) fluoro2-deoxy-D-glucose positron emission tomography/ computed tomography (18F-FDG PET/CT). The ultrasound revealed a solid hypoechoic mass measuring $5 \mathrm{~cm}$ in the right supraclavicular region, identified as lymph nodes. The 18 -FDG-PET/CT revealed a significant tracer uptake in the right supraclavicular region, the anterior upper mediastinum and the left adrenal gland (Figures 1A \& 1B).

At presentation he did not complain of any fever, dizziness, weight loss, abdominal pain or any other symptoms. His blood pressure was $120 / 80 \mathrm{mmHg}$, with a regular heart rate of 75 beats $/ \mathrm{min}$ and body temperature of $36.5^{\circ} \mathrm{C}$. Physical examination confirmed the presence of a painless, well-defined, firm mass in the right supraclavicular region. The adrenals were not palpable. Laboratory tests revealed increased 24 hour urinary dopamine and catecholamine levels as well as serum neuron specific enolase (NSE) (Table 1), suggestive of the presence of a pheochromocytoma.
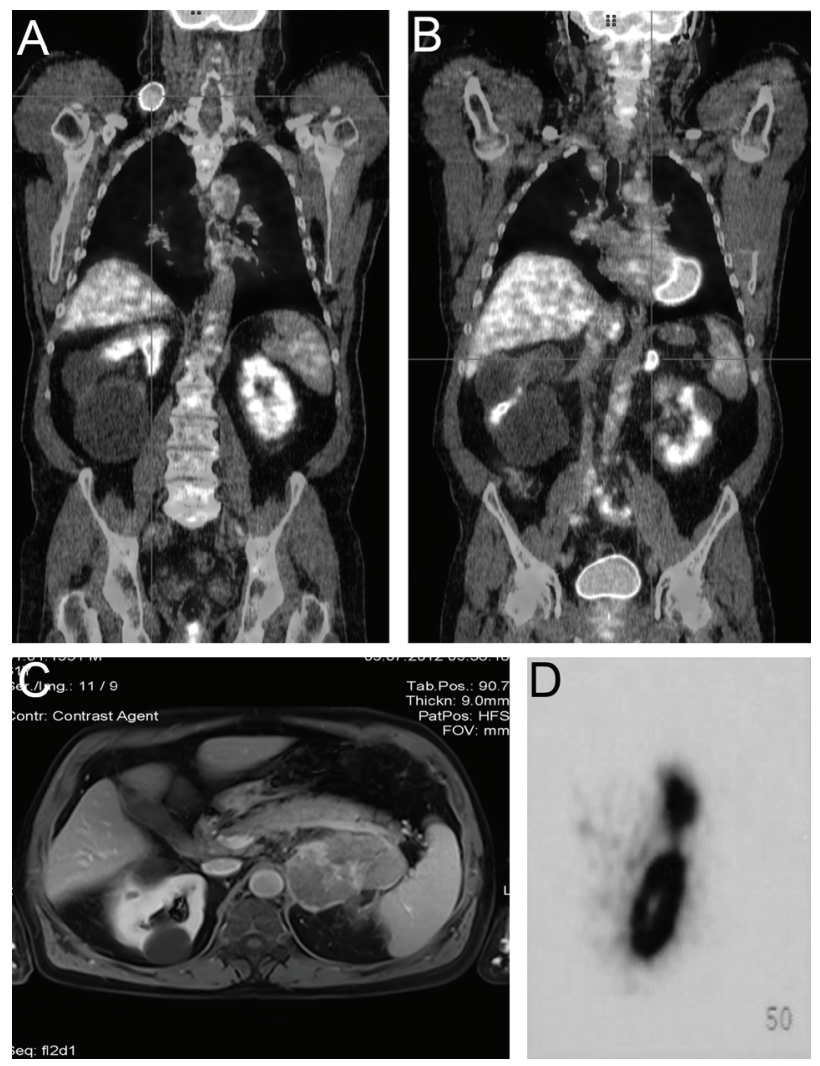

Figure 1. A) $18 \mathrm{~F}-\mathrm{FDG}$ PET/CT: significant tracer uptake in the right supraclavicular region (SUVmax: 10.0). B) Significant tracer uptake in the left adrenal grand (SUVmax: 4.7). C) MRI: large solid mass $88.5 \mathrm{~min}$ in diameter of the left adrenal gland, with inhomogeneous enhancement of the contrast agent and a low diffusion rate. D) OctreoScan: increased trace uptake in the left adrenal gland. 
Increased levels of serum calcitonin were also detected (Table 1).

A computed tomography (CT) scan of the chest confirmed the block of lymph nodes measuring $5.5 \mathrm{~cm}$ in the right supraclavicular area and a large, solid, inhomogeneous mass in the left adrenal gland. Magnetic resonance imaging (MRI) of the abdomen revealed a solid left adrenal mass measuring $88.5 \mathrm{~mm}$, with inhomogeneous contrast enhancement agent and a low diffusion rate (Figure 1C). The mass was identified as a pheochromocytoma. In 111-Pentetreotide (OctreoScan) scintigraphy demonstrated a significantly increased tracer uptake in the right supraclavicular region, the anterior upper mediastinum and the left adrenal gland (Figure 1D). The CT scan of the chest did not show the mass to be in the anterior upper mediastinum as seen on OctreoScan. However, metaiodo-benzyl-guanidine ( ${ }^{131} \mathrm{MIBG}$ ) scintigraphy was negative.

Table 1. Laboratory data

\begin{tabular}{|c|c|c|c|}
\hline & $\begin{array}{c}\text { Reference } \\
\text { range Adults }\end{array}$ & $\begin{array}{c}\text { Before } \\
\text { surgery }\end{array}$ & $\begin{array}{c}\text { After } \\
\text { surgery }\end{array}$ \\
\hline Calcitonin (pg/mL) & $<18$ & 217 & 351 \\
\hline Thyroglobulin $(\mu \mathrm{IU} / \mathrm{ml})$ & $<78$ & 0.26 & \\
\hline Calcium (mg/dl) & $8.4-10.1$ & 8.5 & \\
\hline Parathormone (pg/ml) & $15-65$ & 72 & \\
\hline $25(\mathrm{OH})$ vitamin $\mathrm{D}(\mu \mathrm{g} / \mathrm{L})$ & $25-80$ & 20.9 & \\
\hline $\mathrm{ACTH}(\mathrm{pg} / \mathrm{ml})$ & $6-46$ & 26.7 & \\
\hline Cortisol (nmol/l) & $118-618$ & 326 & \\
\hline DHEA-S $(\mu \mathrm{g} / \mathrm{dl})$ & $51.7-295$ & 93 & \\
\hline $\begin{array}{l}\text { 24h urinary catecholamine } \\
(\mu \mathrm{g} / 24 \mathrm{~h})\end{array}$ & $80-515$ & 817 & \\
\hline $\begin{array}{l}\text { 24h urinary adrenaline } \\
(\mu \mathrm{g} / 24 \mathrm{~h})\end{array}$ & $4-25$ & 8.8 & 10.8 \\
\hline $\begin{array}{l}\text { 24h urinary noradrenaline } \\
(\mu \mathrm{g} / 24 \mathrm{~h})\end{array}$ & $20-105$ & 105.1 & 88.9 \\
\hline $\begin{array}{l}\text { 24h urinary dopamine } \\
(\mu \mathrm{g} / 24 \mathrm{~h})\end{array}$ & $65-400$ & 703 & 332 \\
\hline $24 \mathrm{~h}$ urinary VMA $(\mathrm{mg} / 24 \mathrm{~h})$ & $1-11$ & 6.2 & 3,9 \\
\hline $\begin{array}{l}\text { Neuron Specific Enolase } \\
(\mu \mathrm{g} / 1)\end{array}$ & $<16$ & 79 & 11.9 \\
\hline Chromogranin A (nmol/l) & $<4$ & 2.2 & \\
\hline
\end{tabular}

ACTH: Adrenocorticotropic hormone, DHEA-S: Dehydroepiandrosterone-Sulfate, VMA: Vanilmandelic Acid
Three days prior to the operation, the patient was urgently subjected to a new CT scan due to the sudden onset of abdominal pain. The $\mathrm{CT}$ revealed a significant increase in the adrenal tumor size (from $88.5 \mathrm{~mm}$ to $16.5 \mathrm{~cm}$ ), possibly due to intratumoral hemorrhage. Right supraclavicular lymph nodes dissection and open left adrenalectomy were performed. A well-defined, encapsulated, inhomogeneous mass of the adrenal gland with hemorrhagic and necrotic areas was removed. The tumor was $16.5 \times 10.7 \times 10 \mathrm{~cm}$ in diameter and weighed 761 gr. Intraoperatively, no distant metastasis or infiltration of the surrounding lymph nodes and organs were detected. The patient had an uneventful recovery and was discharged on the seventh postoperative day.

Histologic examination of both the right supraclavicular mass and the adrenal tumor, with hematoxylin \& eosin (H\&E) staining (Figures $2 \mathrm{~A} \& 2 \mathrm{~B}$ ) revealed malignant infiltration with identical histopathological and cytological features. In both specimens a poorly

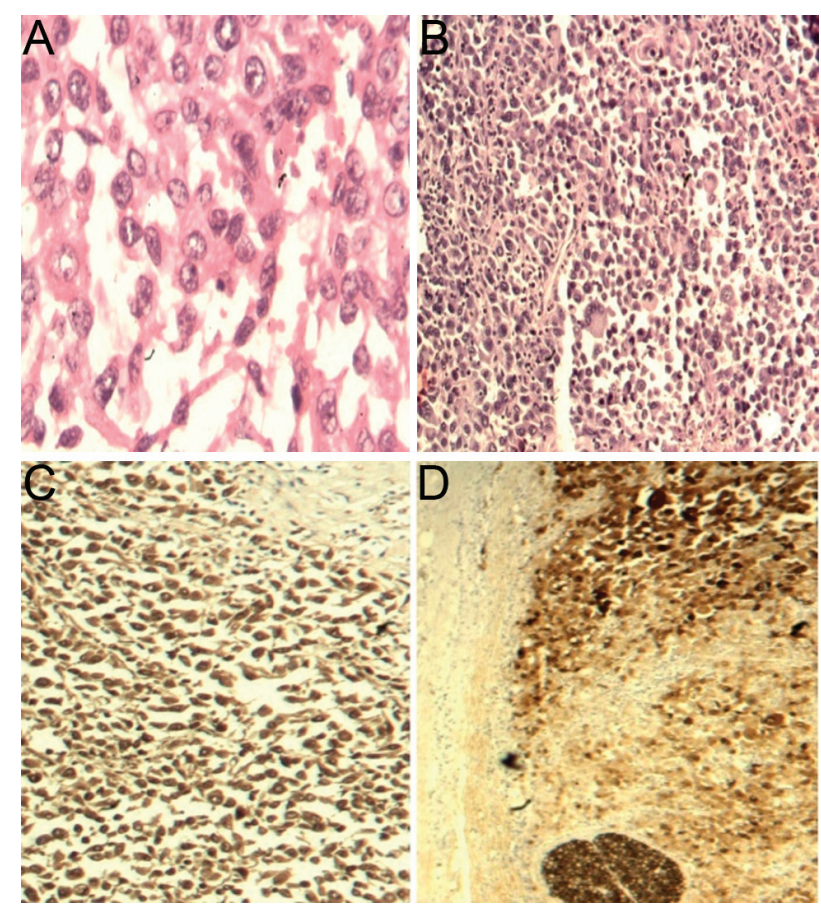

Figure 2. A) Adrenal tumor infiltration with microscopic features of malignant melanoma, in common H\&E stain $(1 \times 400)$. B) Supraclavicular lymph node infiltration with highly anaplastic cells of malignant melanoma in H\&E stain. C \& D) Strong S-100 immunoreactivity of malignant melanoma cells infiltrating the adrenal gland (C) and the neck lymph nodes (D) $(1 \times 100)$. 
differentiated malignant neoplasm was observed, composed of spindle-shaped or epithelioid cells, with eosinophilic cytoplasm, markedly atypical and pleomorphic nuclei with prominent nucleoli, abundant mitoses and areas of necrosis.

Immunohistochemical evaluation of both specimens showed strong positivity for S-100 protein (Figures 2C \& 2D), melanoma-associated protein A (Melan-A), human melanoma black-45 (HMB45), microphthalmia-associated transcription factor (MiTF), tyrosinase and vimentin; immunostaining for cytokeratins, inhibin, synaptophysin and chromogranin, smooth muscle actin and desmin was negative. The strong positivity of S-100 conjunction with the other immunohistochemical markers confirmed the diagnosis of a metastatic, highly aggressive malignant melanoma as per the established criteria. ${ }^{11}$ The diagnosis was confirmed on independent histological review.

The patient was reevaluated following the pathology report. After that he was subjected to a thorough dermatological examination and an endoscopy of the gastrointestinal tract which were all without remarkable findings. Postoperative laboratory tests are shown in Table 1. Serum calcitonin levels remained increased in contrast to dopamine and NSE levels which returned to the normal range. In addition, postoperative OctreoScan did not demonstrate a tracer uptake in the right supraclavicular region and the left adrenal gland, except for a significant tracer uptake in the lymph nodes of the anterior upper mediastinum which were not removed at this stage.

A genetic test for BRAF gene V600E mutation was negative. The patient received chemotherapy with dacarbazine. He died eight months after the initial diagnosis of malignant melanoma and no autopsy was performed.

\section{DISCUSSION}

In this article, we report a rare case of concomitant thyroid carcinoma (papillary and medullary) with a probably primary adrenal malignant melanoma mimicking a pheochromocytoma. Malignant cutaneous melanoma derives from melanocytes normally present in the epidermis. Adrenal glands are usually involved in metastasis from melanoma of the skin or the ocular apparatus (choroid plexus), and less frequently from other tissues like upper respiratory system or gastrointestinal tract. ${ }^{12,13}$

Our patient was entirely asymptomatic at presentation and was taking only levothyroxine. The clinical presentation of pheochromocytomas is due to the release of catecholamines, namely epinephrine, norepinephrine and dopamine into the circulation. Predominance of dopamine excretion has been associated with lack of the characteristic symptoms indicative of catecholamine excess and sporadic cases of either asymptomatic, hypertensive or hypotensive patients have been published. ${ }^{14}$ Dopamine-secreting pheochromocytomas have been associated with higher rates of malignancy. In most cases dopamine-secreting pheochromocytomas are presented as a large adrenal mass causing mass effects and are often already metastatic at presentation. Urine catecholamines and metanephrines have been considered as the golden standard in evaluating a patient suspected for pheochromocytoma. ${ }^{14,15}$ In addition, measurements of methoxytyramine in plasma and urine could be used as a marker of dopamine-producing tumors. ${ }^{16}$

Adrenal melanomas, primary or metastatic, are usually discovered incidentally during routine imaging of the abdomen and are most commonly asymptomatic. Should they exist, signs or symptoms are non-specific and patients manifest pain, fever, nausea, vomiting or abdominal discomfort due to mass effects of the malignancy. ${ }^{17,18}$

The present case had increased serum NSE and 24-hour urinary dopamine levels preoperatively which returned into the normal range after surgery (Table 1). High circulating levels of NSE have been found in patients with various neuroendocrine tumors, including pheochromocytomas (in 50\% of cases) and have been associated with poor tumor differentiation. ${ }^{19} \mathrm{NSE}$ has also been proposed as a serum marker for melanoma, particularly in patients with advanced stage disease. ${ }^{20}$

Our patient had increased serum calcitonin levels three years after total thyroidectomy with central and right lateral cervical lymph node dissection, which means that there was probably a recurrence of medullary thyroid carcinoma. The FDG-PET/CT and OctreoScan revealed a significant tracer uptake in the lymph nodes in the anterior upper mediastinum that were not removed in the surgery. However, it is 
unlikely that these lymph nodes represent a metastasis of medullary thyroid carcinoma. The sensitivity of FDG PET/CT is extremely variable because it is strictly correlated as much with the tumor's size, its proliferation and its differentiation index as with serum calcitonin levels. ${ }^{21}$ FDG-PET/CT has a 78\% sensitivity with serum calcitonin levels above 1000 $\mathrm{pg} / \mathrm{ml}$ and $20 \%$ in patients with serum calcitonin levels around $500 \mathrm{pg} / \mathrm{ml}$. MIBG scintigraphy, which was negative in our patient, was also used for the detection of metastatic medullary thyroid carcinoma but with poor diagnostic accuracy due to a very low sensitivity rate around $25-30 \%$ and a high specificity value around $95 \%$. OctreoScan was also used for the detection of medullary thyroid carcinoma with sensitivity values ranging from $37 \%$ to $75 \%$ because of the inhomogeneous distribution of somatostatin receptors in MTC tissue and their lower levels. ${ }^{22}$ Our patient had serum calcitonin levels ranging from $217 \mathrm{pg} / \mathrm{ml}$ before surgery to $351 \mathrm{pg} / \mathrm{ml}$ after surgery, negative MIBG and positive OctreoScan and FDG $\mathrm{PET} / \mathrm{CT}$ for the adrenal and the suppraclavicular lesions which were identified in histological examination as malignant melanomas. OctreoScan and FDG $\mathrm{PET} / \mathrm{CT}$ which were positive for the lymph nodes in the anterior upper mediastinum and not removed in the surgery performed were more suggestive of malignant melanoma.

Catecholamine synthesis in melanoma seems to be related to the neural crest origin of melanoma cells. Chromaffin cells of the medulla and melanocytes have a common embryogenesis from the neural crest, derived from embryonic neuroectoderm (neural tube epithelium), explaining the development of primary melanoma in the adrenal gland. ${ }^{23}$ In human melanoma cell lines increased levels of catecholamines are produced. ${ }^{24}$ Melanomas produce dopamine, serotonin and adrenaline. ${ }^{23,24}$

In patients with malignant melanoma, high levels of 3,4-dihydroxyphenylalanine (DOPA) and dopamine are excreted in urine, especially at more advanced stages of the disease..$^{25}$ Tyrosine is derived from hydroxylation of phenylalanine. Normal and malignant melanocytes express the enzyme tyrosine hydroxylase, a copper-containing enzyme, that catalyzes the first steps of the biosynthetic pathway of melanins from tyrosine initially and DOPA subsequently, leading to conversion of the latter to dopaquinone (a melanin precursor). DOPA is oxidized to dopaquinone by DOPA oxidase, an enzyme occurring in melanin granules. ${ }^{25,26}$

In melanoma tissue there is induction of tyrosine hydroxylase leading to production of DOPA. Activity of DOPA decarboxylase converts part of DOPA to 3,4-dihydroxyphenylethylamine (dopamine, DA). The pattern of excretion shows a wide variation among patients possibly due to modifications in structure or the enzymatic potency of tyrosine hydroxylase in melanoma tissue. ${ }^{25}$ High urinary dopamine and melanuria in patients with adrenal melanoma have been described. ${ }^{26}$ The biosynthetic pathway of melanins and catecholamines is presented in Figure 3.

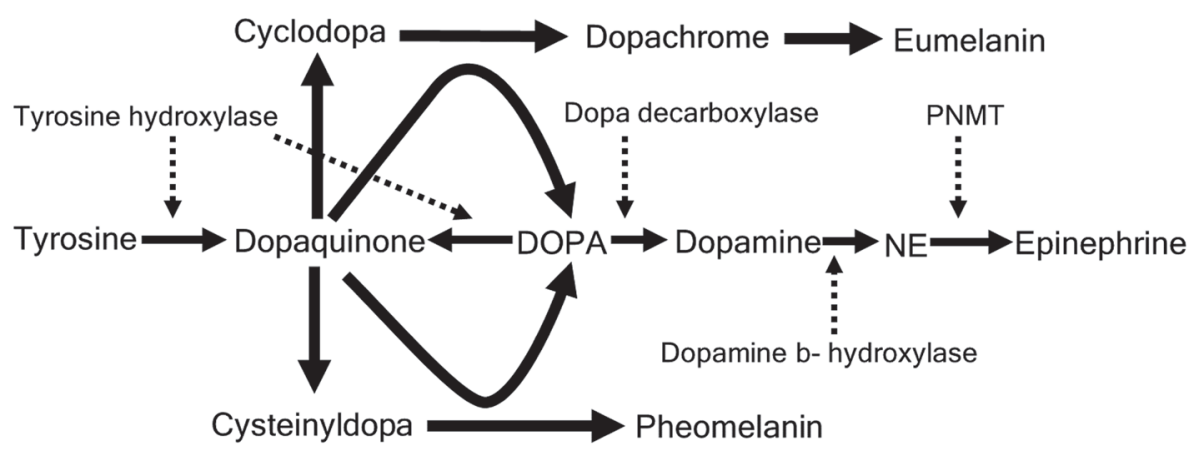

Figure 3. Biosynthetic pathway of melanins and catecholamines. Normal and malignant melanocytes express the enzyme tyrosine hydroxylase, a copper-containing enzyme that catalyzes the first steps of biosynthetic pathway of melanins from tyrosine. In melanoma tissue there is induction of tyrosine hydroxylase leading to production of DOPA. Activity of DOPA decarboxylase converts part of DOPA to 3,4-dihydroxyphenylethylamine (dopamine, DA) (for details refer to text). NE: Norepinephrine, PNMT: Phenylethanolamine $\mathrm{N}$-Methyl Transferase. Dotted arrow indicates position of enzymatic action. 
The CT scan revealed a large, solid, inhomogeneous adrenal mass. Then, on MRI scan an $88.5 \mathrm{~mm}$ solid mass with inhomogeneous enhancement of the contrast agent and low diffusion rate was confirmed. Generally, on CT scan, large pheochromocytomas ( $>2$ $3 \mathrm{~cm}$ ) can be inhomogeneous with areas of hemorrhage and low attenuation necrosis. On MRI, after contrast administration, pheochromocytomas enhance avidly and have a prolonged washout phase, at least in their viable areas, while necrotic parts of the tumor do not enhance. ${ }^{27}$ Adrenal metastases are usually presented with similar imaging features on MRI as well. ${ }^{28}$

Another imaging diagnostic tool for the detection of neuroendocrine tumors is somatostatin receptor scintigraphy. Our patient had a positive OctreoScan. Pheochromocytomas sometimes express somatostatin receptors, but most of the times benign tumors are octreotide-negative (66\%-75\%), even though they appear MIBG-positive. However, octreotide scintigraphy has $90 \%$ sensitivity in detecting malignant-metastatic pheochromocytomas and paragangliomas. ${ }^{27,29}$ Similarly, octreotide scintigraphy showed a sensitivity and specificity of $87 \%$ and $94 \%$, respectively, for diagnosing metastases from malignant melanomas..$^{30}$ OctreoScan was also used for the detection of medullary thyroid carcinoma with sensitivity values ranging from $37 \%$ to $75 \%$, because of the inhomogeneous distribution of somatostatin receptors in MTC tissue and their lower levels. ${ }^{19}$ An OctreoScan that was positive for the lymph nodes in the anterior upper mediastinum not removed in the surgery performed was more suggestive of malignant melanoma rather than a metastasis of medullary thyroid carcinoma.

Our patient's I ${ }^{131}$ MIBG scanning was negative. MIBG is a noradrenaline analogue that is taken up by pheochromocytoma cells using the noradrenaline transporting system, and, when radiolabelled with I ${ }^{131}$ or $\mathrm{I}^{123}$, it constitutes an imaging technique traditionally considered to be specific for the localization of pheochromocytomas. However, $\mathrm{I}^{131} \mathrm{MIBG}$ has $77-90 \%$ sensitivity and $95-100 \%$ specificity in detecting such tumors. ${ }^{31}$ False negative results have been associated with dedifferentiation of the tumor as well as with exclusively dopamine-secreting pheochromocytomas for which the sensitivity drops to a level of $66 \%{ }^{32}$

The differential diagnosis between benign and malignant lesions of the adrenal glands is not always easy. FDG-PET/CT provides both anatomical and functional information and the maximum standardized uptake value $\left(\mathrm{SUV}_{\text {max }}\right)$ is the best index to assess disease activity. An SUV max $_{\text {max }}$ cut-off value of 4.2 corresponds to a sensitivity of $88.6 \%$ and specificity of $88.2 \%$ in detecting malignant adrenal lesions. ${ }^{33}$ Notably, our patient had an $\mathrm{SUV}_{\max }$ of 4.7 in his adrenal mass, suggesting the presence of malignancy.

Our patient had a history of a multifocal papillary thyroid carcinoma concurrent with an adrenal malignant melanoma. There is a high prevalence of BRAF (V600E) activating mutations in papillary thyroid carcinoma, cutaneous malignant melanoma and hairy cell leukemia. Recent studies in the clinical trials of BRAF inhibitors in patients with malignant melanoma are promising for the treatment of this highly lethal disease. ${ }^{34}$ Of note, a genetic test for BRAF (V600E) activating mutations was negative in our patient.

Adrenal glands are usually involved in metastasis from melanoma of the skin or the ocular apparatus (choroid plexus) and less frequently from other tissues like the upper respiratory system or gastrointestinal tract. ${ }^{14,17}$ In rare cases, adrenal melanomas can occur either as primary tumors, or as metastatic, but of unknown primary origin, in which case differential diagnosis before the pathology examination is impossible. Several rigid diagnostic criteria have been established for defining an adrenal melanoma as primary: i) malignant involvement of a single adrenal gland; ii) absence of another malignant melanoma site; iii) no previous excisions of pigmented mucous, cutaneous or eye lesions; and iv) exclusion of any hidden primary lesion preferably detected at autopsy. ${ }^{35}$ Our patient reported the excision of an epidermal nevus, three years before presentation, which was not subjected to histological examination, after the reassurance of his dermatologist that it was non-significant. He also reported that a few months earlier he had been subjected to an ophthalmological examination which was normal. Clinical examination did not reveal any primary site of melanoma since the abovementioned dermatological examination and endoscopy of the gastrointestinal tract were without remarkable findings. In respect of the above rigid diagnostic criteria for accepting an adrenal melanoma as primary, our patient had a score of 3 to 4 . 
In conclusion, this rare case illustrates the difficulties in the differential diagnosis of a dopaminesecreting adrenal mass. Both adrenal melanomas and pheochromocytomas should be considered in the differential diagnosis of an adrenal mass, especially when the primary biochemical finding is increased urine dopamine excretion, in order to determine the best diagnostic approach for the patient and select the most appropriate surgical management.

\section{REFERENCES}

1. Wohllk N, Schweizer H, Erlic Z, et al, 2010 Multiple endocrine neoplasia type 2. Best Pract Res Clin Endocrinol Metab 24: 371-377.

2. Ng VW, Ma RC, So WY, et al, 2010 Evaluation of functional and malignant adrenal incidentalomas. Arch Intern Med 170: 2017-2020.

3. Zeiger MA, Siegelman SS, Hamrahian AH, 2011 Medical and Surgical Evaluation and Treatment of Adrenal Incidentalomas. J Clin Endocrinol Metab 96: 2004-2015.

4. Muth A, Persson F, Jansson S, et al, 2010 Prognostic factors for survival after surgery for adrenal metastasis. Eur J Surg Oncol 36: 699-704.

5. Bradley CT, Strong VE, 2014 Surgical management of adrenal metastasis. J Surg Oncol 109: 31-35.

6. Hofmann U, Szedlak M, Rittgen W, Jung EG, Schadendorf D, 2002 Primary staging and follow-up in melanoma patients - monocenter evaluation of methods, costs and patient survival. Br J Cancer 87: 151-157.

7. Atallah E, Flaherty L, 2005 Treatment of metastatic malignant melanoma. Curr Treat Options Oncol 6: 185-193.

8. Avgerinos DV, Nazarian M, Scherr DS, Girardi LN, 2012 Primary adrenal melanoma with inferior vena cava thrombus. Ann Thorac Surg 94: 2108-2110.

9. Ejaz S, Shawa H, Henderson SA, Habra MA, 2013 Melanoma of unknown primary origin presenting as a rapidly enlarging adrenal mass. BMJ Case Rep 19: 2013. pii: bcr2013009727.

10. Gonzalez-Saez L, Pita-Fernandez S, Lorenzo-Patino M, Arnal-Monreal F, Machuca-Santacruz J, RomeroGonzalez J, 2011 Primary melanoma of the adrenal gland: a case report and review of the literature. J Med Case Rep 5: 273.

11. Ohsie SJ, Sarantopoulos GP, Cochran AJ, Binder SW, 2008 Immunohistochemical characteristics of melanoma. J Cutan Pathol 35: 433-444.

12. Granero LE, Al-Lawati T, Bobin J-Y, 2004 Primary Melanoma of the Adrenal Gland, a Continuous Dilemma: Report of a Case. Surg Today 34: 554-556.

13. Castillo OA, Vitagliano G, Kerkebe M, Parma P, Pinto I, Diaz M, 2007 Laparoscopic Adrenalectomy for Suspected Metastasis of Adrenal Glands: Our Experience. Urology 69: 637-641.
14. Poirier É, Thauvette D, Hogue JC, 2013 Management of Exclusively Dopamine-Secreting Abdominal Pheochromocytomas. J Am Coll Surg 216: 340-346.

15. Dubois LA, Gray DK, 2005 Dopamine-secreting pheochromocytomas: in search of a syndrome. World J Surg 29: 909-913.

16. Eisenhofer G, Goldstein DS, Sullivan P, et al, 2005 Biochemical and clinical manifestations of dopamineproducing paragangliomas: utility of plasma methoxytyramine. J Clin Endocrinol Metab 90: 2068-2075.

17. Collinson FJ, Lam TK, Bruijn WMJ, et al, 2008 Longterm Survival and Occasional Regression of Distant Melanoma Metastases after Adrenal Metastasectomy. Ann Surg Oncol 15: 1741-1749.

18. Lo CY, Van Heerden JA, Soreide JA, et al, 1996 Adrenalectomy for metastatic disease to the adrenal glands. Br J Surg 83: 528-531.

19. Baudin E, Gigliotti A, Ducreux M, et al, 1998 Neuronspecific enolase and chromogranin A as markers of neuroendocrine tumours. Br J Cancer 78: 1102-1107.

20. Tofani A, Cioffi RP, Sciuto R, et al, 1997 S-100 and NSE as serum markers in melanoma. Acta Oncol 36: 761-764.

21. Sisson JC, Yanik GA, 2012 Theranostics: evolution of the radiopharmaceutical meta-iodobenzylguanidine in endocrine tumors. Semin Nucl Med 42: 171-184.

22. Castellani MR, Seregni E, Maccauro M, et al, 2008 MIBG for diagnosis and therapy of medullary thyroid carcinoma: is there still a role? Q J Nucl Med Mol Imaging 52: 430-440.

23. Tanas MR, Rubin BP, 2009 Malignant neuroectodermal tumor with melanocytic and rhabdomyoblastic differentiation. Rare Tumors 1(2) e26 doi: 10.4081/rt.2009.e26.

24. McEwan M, Parsons PG, 1987 Inhibition of Melanization in Human Melanoma Cells by a Serotonin Uptake Inhibitor. J Invest Dermatol 89: 82-86.

25. Hinterberger H, Freedman A, Barthoeomew RJ, 1972 Precursors of melanin in the blood and urine in malignant melanoma. Clin Chim Acta 9: 395-400.

26. Voorhess ML, 1970 Urinary excretion of DOPA and metabolites by patients with melanoma. Cancer 26: 146-149.

27. Mackenzie IS, Ashby MJ, Donovan T, Voutnis DD, Brown MJ, 2006 Bilateral adrenal masses: pheochromocytoma or melanoma? J R Soc Med 99: 153-155.

28. Leung K, Stamm M, Raja A, Low G, 2013 Pheochromocytoma: the range of appearances on ultrasound, CT, MRI, and functional imaging. AJR Am J Roentgenol 200: 370-378.

29. Van Berkel A, Pacak K, Lenders JW, 2014 Should every patient diagnosed with a pheochromocytoma have a ${ }^{123} \mathrm{I}-$ MIBG scintigraphy? Clin Endocrinol (Oxf) 81: 329-333.

30. Zissimopoulos A, Karpouzis A, Kouskoukis C, 2009 Iotandium-111 pentetreotide scintigraphy and CT scans after 3 years in the follow-up of patients with malignant melanoma. Hell J Nucl Med 12: 142-145. 
31. Ilias I, Sahdev A, Reznek RH, Grossman AB, Pacak K, 2007 The optimal imaging of adrenal tumours: a comparison of different methods. Endocr Relat Cancer 14: 587-599.

32. Proye C, Fossati P, Fontaine P, et al, 1986 Dopaminesecreting pheochromocytoma: an unrecognized entity? Classification of pheochromocytomas according to their type of secretion. Surgery 100: 1154-1162.

33. Ozcan Kara P, Kara T, Kara Gedik G, et al, 2011 The role of fluorodeoxyglucose positron emission tomography/computed tomography in differentiating between benign and malignant adrenal lesions. Nucl Med Commun 32: 106-112.

34. Ziai J, Hui P, 2012 BRAF mutation testing in clinical practice. Expert Rev Mol Diagn 12: 127-138.

35. Carstens PHB, Kuhns JG, Ghazi C, 1984 Primary malignant melanomas of the lung and adrenal. Hum Pathol 15: $910-914$. 